\title{
Entéropathies immunes et auto-immunes
}

\author{
Philip M. Sherman ${ }^{a}$ Ernest Cutz $^{a}$ Olivier Goulet ${ }^{\mathrm{b}}$ \\ ${ }^{a}$ Hospital for Sick Children, University of Toronto, Toronto, Canada; \\ ${ }^{\mathrm{b}}$ Groupe Hospitalier Necker-Enfant Malade, Paris, France
}

\section{Mots-clés}

Diarrhée, enfants et nourrissons - Entéropathie, immune et auto-immune - Syndrome IPEX .

Auto-immunité

\section{Résumé}

L'entéropathie auto-immune débute typiquement par une diarrhée prolongée au cours de la première année de la vie. Des anomalies sous-jacentes des fonctions immunitaires, y compris des lymphocytes $T$ régulateurs, doivent être exclues. Le traitement associe la réhabilitation nutritionnelle, l'immunosuppression et, dans certains cas, la greffe de moelle.

Copyright (c) 2006 Nestec Ltd., Vevey/S. Karger AG, Basel

\section{Introduction}

L'entéropathie auto-immune et les entéropathies dues à des déficits immunitaires primitifs sont des causes relativement peu fréquentes de diarrhée prolongée du nourrisson. Face à un nourrisson ou un jeune enfant présentant une diarrhée chronique inexpliquée, les diagnostics différentiels sont très nombreux [1] et différents de ceux qui se poseraient chez l'adulte [2, 3]. Dans la plupart des pays du monde, les entéropathies infectieuses et postinfectieuses demeurent les causes les plus fréquentes de diarrhée prolongée [4]. Certains auteurs soutiennent que les entéropathies allergiques sont fréquentes aussi chez les jeunes enfants, même en l'absence de manifestations d'atopie ou d'antécédents familiaux d'allergie. Plus souvent, une colite allergique induite par les protéines du lait ou du soja peut survenir chez des nouveau-nés et des nourrissons, même nourris uniquement au sein. Chez l'enfant plus âgé ayant reçu du gluten dans son alimentation, une maladie coeliaque peut être évoquée, car il est de plus en plus clair qu'elle peut se manifester de façon très variable et elle peut être traitée efficacement par un régime d'exclusion [5].

Une façon utile d'aborder les étiologies possibles de ces diarrhées intraitables est de distinguer celles dans lesquelles l'unité villosité-crypte est relativement intacte de celles où l'architecture de cette même unité est anormale (il s'agit alors d'une entéropathie). Dans le premier cas, il faut envisager des affections telles que des anomalies congénitales du transport ionique ou une diarrhée sécrétoire, due à des entérotoxines ou des tumeurs hormonosécrétantes [1]. En revanche, une entéropathie avec réduction de la hauteur des villosités doit évoquer un ensemble totalement différent de diagnostics. Après l'exclusion d'étiologies infectieuses et allergiques, une diarrhée chronique profuse débutant au cours des tout premiers jours de la vie doit faire envisager un diagnostic de maladie des inclusions microvillositaires [6] également appelée atrophie microvillositaire familiale [7] ou syndrome de Davidson.

\begin{tabular}{ll}
\hline KARGER & ( ) 2006 Nestec Ltd., Vevey/S. Karger AG, Basel \\
0250-9644/06/0641-0007\$23.50/0 \\
$\begin{array}{l}\text { Fax +4161306 1234 } \\
\begin{array}{l}\text { E-Mail karger@karger.ch } \\
\text { www.karger.com }\end{array}\end{array}$ & $\begin{array}{l}\text { Accessible en ligne à: } \\
\text { www.karger.com/anf }\end{array}$
\end{tabular}


La dysplasie épithéliale intestinale [8], également appelée «tufting enteropathy» («entéropathie à houpettes»), peut se présenter de façon très similaire à une entéropathie auto-immune, sa diarrhée chronique étant d'apparition légèrement plus tardive [9]. Il est intéressant de noter que de nombreux nourrissons européens et nord-américains chez lesquels cette entéropathie a été décrite étaient issus de familles originaires d'Afrique du Nord, de Turquie et, particulièrement, de l'Ile de Malte. En conséquence, une dysplasie épithéliale intestinale doit certainement être sérieusement evoquée chez les nourrissons originaires de ces regions.

\section{Présentation clinique}

Les premières manifestations d'une entéropathie autoimmune apparaissent typiquement après la période néonatale, au cours de la première année de la vie. Des antécédents de polyhydramnios maternel au cours de la grossesse et/ou la présence d'autres enfants atteints dans la famille doivent susciter la recherche d'autres causes de diarrhée prolongée à début néonatal comme, par exemple, une diarrhée chlorée congénitale [1].

La plupart des cas d'entéropathie auto-immune débutent par une diarrhée chronique aqueuse sans épisode de rémission apparaissant à l'âge de 2 à 4 mois après une grossesse et une période néonatale sans complications. Un début plus tardif des troubles est toutefois classique par une diarrhée isolée à l'adolescence [10] ou à l'âge adulte [11]. Un diagnostic de sprue réfractaire est typiquement posé dans ces cas à début tardif, jusqu'à la recherche d'anticorps anti-entérocytes. On ignore si ces cas à début tardif sont dus à la même anomalie moléculaire que ceux dont la diarrhée est apparue au cours de la première année de la vie.

Bien que ces affections auto-immunes se manifestent principalement par des entéropathies, le côlon est souvent également atteint. Des signes et symptômes de colite (par exemple défécation impérieuse, ténesme, présence de sang frais et de mucus dans les selles) accompagnant une diarrhée prolongée ne répondant pas à un essai de diète élémentaire doivent faire suspecter une entéropathie auto-immune, en particulier s'il existe aussi un ralentissement de la croissance et une malnutrition.

L'existence d'autres affections auto-immunes accroît également le risque d'entéropathie auto-immune. Les premiers cas d'entéropathie auto-immune décrits dans la littérature scientifique étaient associés à un diabète de type 1 , une thyroïdite auto-immune, une anémie hémo- lytique auto-immune ou des affections cutanées qui avaient probablement une base auto-immune. La plupart des cas décrits étaient également associés à une néphropathie tubulaire.

\section{Observations anatomopathologiques}

Les biopsies de l'intestin grêle sont particulièrement utiles chez les nourrissons et les enfants présentant une diarrhée prolongée. Les résultats des examens morphologiques et immunohistochimiques sont non seulement utiles pour établir un diagnostic spécifique, mais aussi pour exclure d'autres affections quand ces explorations ne sont pas concluantes [12].

En cas d'entéropathie auto-immune, les aspects observés en microscopie optique (fig. 1a) ressemblent beaucoup à ceux d'une maladie cœliaque même en l'absence d'une épreuve de réintroduction du gluten. Les examens de coupes colorées à l'hématoxyline-éosine révèlent souvent une atrophie totale des villosités et une hyperplasie des cryptes. De plus, la lamina propria de la muqueuse intestinale est le siège d'une importante infiltration de cellules immunitaires, dont des lymphocytes $\mathrm{T}$ activés [13]. L'épithelium peut être infiltré de lymphocytes intraépithéliaux, mais pas au même degré que dans une atrophie villositaire totale due à une maladie coeliaque [14]. L'étude immunohistochimique au moyen d'anticorps spécifiques révèle une importante infiltration de lymphocytes CD3+ dans la lamina propria (fig. 1b). Des données récentes indiquent qu'une cytokine, l'interleukine 21, pourrait médier l'activation des lymphocytes $\mathrm{T}$, altérer la survie des cellules et finalement prédisposer l'hôte affecté à des maladies auto-immunes [15].

L'examen de biopsies coliques montre également des signes d'infiltration de la lamina propria par des cellules immunitaires chez les enfants atteints d'une entéropathie auto-immune. Cette infiltration n'est pas observée au cours de diarrhées prolongées relevant d'une autre cause et affectant des nourrissons chez lesquels il n'existe pas de signes d'activation immunitaire en d'autres sites que l'intestin grêle [14].

\section{Auto-anticorps}

La présence d'anticorps sériques d'isotype $\mathrm{G}$, circulant habituellement à un faible titre, se liant à la face apicale des entérocytes est un signe capital d'entéropathie autoimmune (fig. 2). Dans une étude de 25 enfants présentant 
Fig. 1. a Biopsie de la muqueuse de l'intestin grêle d'un enfant présentant une diarrhée prolongée. La biopsie montre une atrophie villositaire totale avec hyperplasie des cryptes et infiltration de la lamina propria par diverses cellules inflammatoires chroniques. HE. Grossissement original approximatif: 20 fois. b Un examen immunohistochimique au moyen d'un anticorps anti-CD3 démontre une infiltration de la lamina propria par de nombreux lymphocytes CD3+. Grossissement original approximatif: 20 fois.
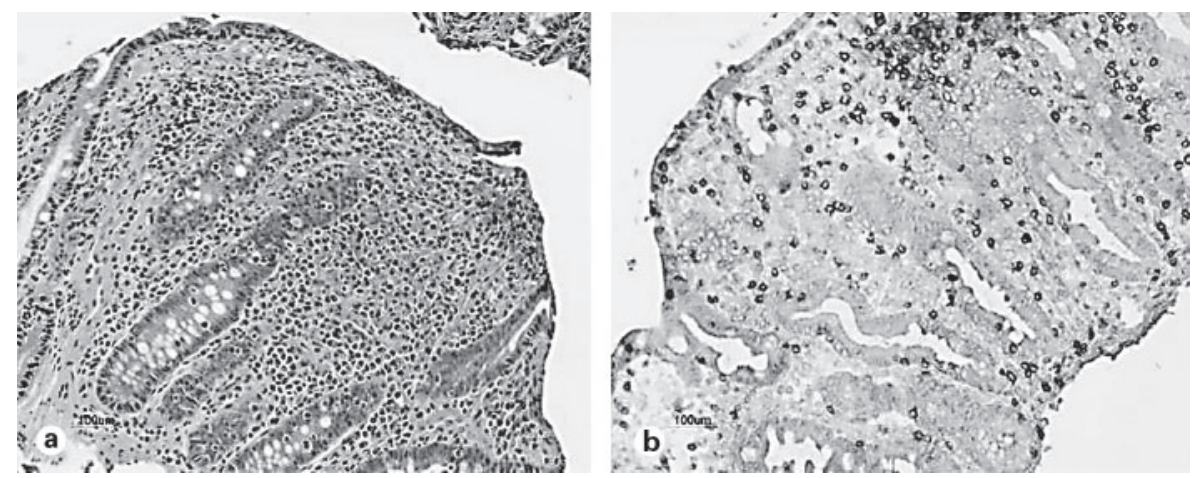

Fig. 2. Un examen en immunofluorescence indirecte démontre la présence d'anticorps anti-entérocytes dans le sérum d'un enfant atteint d'une entéropathie auto-immune. Le marquage par fluorescence dessine la surface apicale des entérocytes le long de l'unité villosité-crypte.

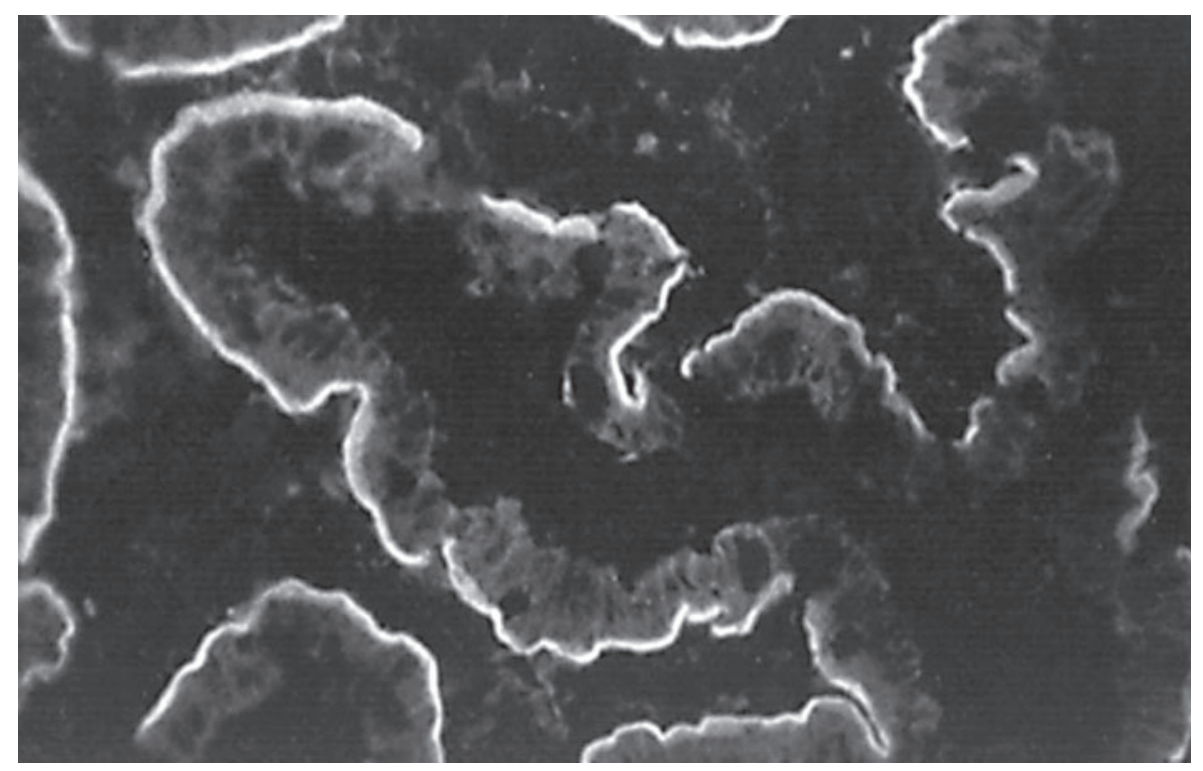

une diarrhée chronique inexpliquée, des anticorps antientérocytes ont été détectés chez 14 (56\%) sujets, alors que des résultats positifs n'étaient observés que chez 3 patients sur $53(5 \%)$ atteints d'une maladie inflammatoire chronique intestinale, 0 sur 10 présentant une maladie cœliaque, et chez aucun enfant sur 50 présentant d'autres affections extradigestives [16]. L'anticorps n'est pas spécifique d'un épitope des entérocytes humains, car les auto-anticorps se lient également aux cellules épithéliales de l'intestin grêle chez le rat, la souris et le cobaye. L'antigène est également détecté dans l'épithélium rénal.

Une caractérisation par Western blot a montré que les autoantigènes sont de 55 [17] ou $75 \mathrm{kDa}[18,19]$ L'autoantigène de $75 \mathrm{kDa}$ est exprimé dans l'intestin grêle, le côlon, le pancréas et les reins [20]. Avec le traitement (envisagé plus bas), les titres d'anticorps anti-entérocytes circulants diminuent ou disparaissent progressivement et totalement, tandis que la situation clinique s'améliore. Les titres des autoanticorps systémiques permettent donc de suivre la réponse aux traitements [17].

Bien que les cibles moléculaires des anticorps anti-entérocytes aient été décrites, certains auteurs estiment que ce test n'est pas suffisamment spécifique. Par exemple, Martin-Villa et coll. [21] ont noté la présence d'anticorps de ce type dans la circulation de sujets n'ayant pas d'atrophie villositaire ni d'antécédents de diarrhée. De ce fait, comme tout autre examen de dépistage, cette recherche d'anticorps anti-entérocytes doit être placée dans les contextes cliniques et pathologiques appropriés.

Dans quelques cas une entéropathie auto-immune avec auto-anticorps circulants, dirigés contre la lignée des cellules caliciformes et non contre les régions apicales des entérocytes, a été décrite [22]. Les biopsies intestinales montrent alors une raréfaction des cellules caliciformes, de Paneth et entéroendocrines [23]. Cet aspect pourrait 
traduire un déficit en un facteur de transcription, Math1 [24]. Par ailleurs, les tableaux cliniques et les constatations pathologiques sont semblables à ceux observés dans les autres cas d'entéropathie auto-immune. Ces données confortent le point de vue selon lequel les lymphocytes $T$ infiltrantes, et non les anticorps systémiques, jouent un rôle dans la physiopathologie de la maladie. Ainsi, les auto-anticorps sont probablement des marqueurs d'une affection sous-jacente déclenchée par une inflammation et des lésions de la muqueuse, mais ne paraissent pas impliqués dans la pathogenie de la maladie $[13,14]$.

\section{Troubles liés à un déficit immunitaire primitif}

Diverses anomalies immunitaires d'origine génétique peuvent s'accompagner de lésions intestinales responsables d'une entéropathie, d'une malabsorption et d'une diarrhée prolongée [25]. Par exemple, la granulomatose septique et la glycogènose du type $1 \mathrm{~b}$ ont été toutes deux associées à une inflammation muqueuse sur toute la longueur du tractus gastro-intestinal, avec des aspects cliniques, radiologiques et endoscopiques pouvant simuler ceux observés dans les maladies inflammatoires chroniques de l'intestin. La gastro-entérite à éosinophiles peut être evoquée comme diagnostic différentiel.

Il est désormais clair qu'il existe des causes multiples au tableau appelé entéropathie auto-immune. Ainsi, des publications indiquent que des déficits primitifs en lymphocytes $\mathrm{T}$ ont des caractéristiques de maladie auto-immune. Arniaz-Villena et coll. [26] ont décrit le cas d'une famille dans laquelle existait une anomalie congénitale de l'expression de la sous-unité du complexe récepteur CD3- $\gamma$ des lymphocytes $T$. Le cas index présentait une diarrhée prolongée associée à des auto-anticorps dirigés contre les cellules épithéliales intestinales, les muscles lisses et les mitochondries ainsi qu'une anémie hémolytique et un déficit sélectif en sous-classe IgG2.

Les cas de deux enfants présentant une dysplasie immuno-osseuse de Schimke et une anomalie de la fonction immunitaire des lymphocytes T ont été publiés [27, 28]. Ces deux enfants avaient une diarrhée chronique et une symptomatologie compatible avec une entéropathie autoimmune, mais aucun anticorps n'a été décelé et des mycobactéries atypiques ont été identifiées dans la muqueuse de l'intestin grêle chez l'un d'entre eux [28]. Cette observation souligne l'importance d'exclure une infection chronique et un déficit immunitaire chez un enfant présentant une diarrhée prolongée inexpliquée.

\section{Syndrome IPEX}

Le syndrome IPEX (dérèglement Immunitaire, Polyendocrinopathie, Entéropathie, liés à l'X) est la cause la mieux caractérisée d'entéropathie auto-immune. Cette maladie est due à des mutations du gène Foxp 3 situé dans la région péricentromérique du chromosomeX:Xp11.23Xp13.3 [29, 30]. Foxp3 (encore appelé JM2 et scurfin) est un facteur de transcription capital pour le contrôle du développement normal des lymphocytes $\mathrm{T}$ régulateurs $[31,32]$. Les mutations entraînent la disparition du contrôle normal des lymphocytes T CD25+, et il existe une activation inappropriée et une dissémination de cellules immunitaires activées à de nombreux organes, dont le tractus intestinal (fig. 3).

Plusieurs mutations du gène FoxP 3 responsables de maladies chez l'homme ont été décrites. Il existe probablement une relation entre génotype et phénotype expliquant les différences d'expression clinique, de sévérité de la maladie, de nombre de manifestations auto-immunes et de pronostic [35]. De fait, l'entéropathie auto-immune peut être subdivisée en 3 groupes: les cas dus à un IPEX, les cas touchant les garçons mais sans mutation du gène FoxP3 et les cas touchant les filles [36].

Les nourrissons de sexe masculin atteints d'un syndrome IPEX présentent un diabète de type 1, une thyroïdite auto-immune, une anémie hémolytique auto-immune (positivité du test de Coombs direct) et diverses lésions cutanées, d'eczéma, de dermatite ichtyosiforme, ou psoriasique, voire une calvitie totale (alopecia universalis) [37]. Le premier signe d'une entéropathie auto-immune est souvent une diarrhée prolongée profuse. Ces enfants peuvent également présenter des manisfestations d'atopie comme des allergies alimentaires, une éosinophilie périphérique et une augmentation de la concentration en immunoglobulines E [38]. Des cas publiés antérieurement d'entéropathie auto-immune survenus chez des garçons avec auto-anticorps dirigés contre d'autres organes que l'intestin et associés à des maladies auto-immunes, doivent être considérés comme des cas possibles de syndrome IPEX (en particulier quand plusieurs membres de la fratrie étaient atteints).

\section{Considérations thérapeutiques}

La prise en charge initiale des nourrissons présentant une diarrhée chronique et chez lesquels une entéropathie auto-immune est envisageable est exactement la même que celle de tous les autres sujets atteints. L'objectif initial 
Fig. 3. Une coupe histologique d'intestin grêle chez un enfant atteint d'un syndrome IPEX montre des signes d'atrophie villositaire totale et l'inflammation importante de la lamina propria avec un infiltrat chronique de cellules inflammatoires.

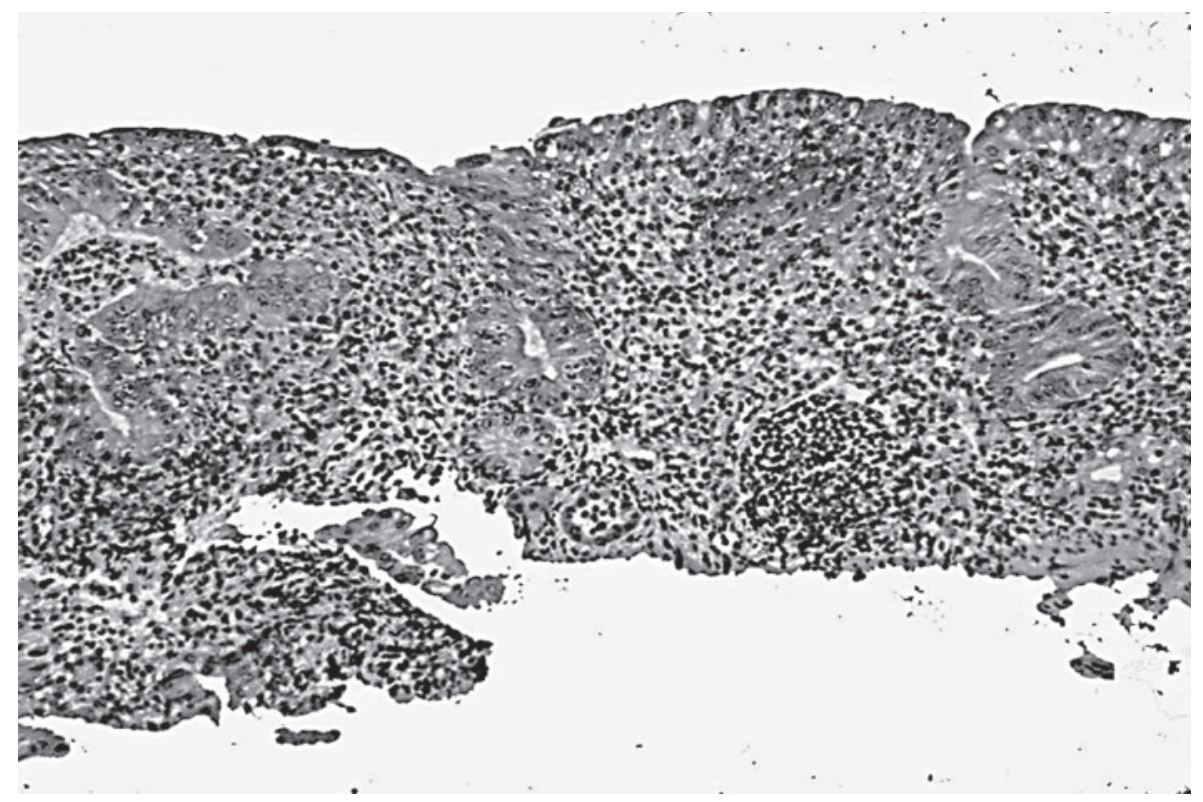

doit être le maintien de la volémie et la renutrition, qui nécessite généralement une nutrition entérale élémentaire ou une nutrition parentérale totale. Ces enfants sont souvent insuffisamment hydratés et alimentés lors des explorations diagnostiques étendues réalisées en milieu hospitalier. Cette approche est déplorable : l'apport de liquide et de nutriments est l'une des priorités [39]. Ce n'est qu'après l'avoir satisfaite que le professionnel de santé pourra considérer la longue liste des éléments à prendre en compte pour le diagnostic différentiel.

L'entéropathie auto-immune répond généralement bien à une puissante immunosuppression. Une corticothérapie générale à forte dose réduit fréquemment l'inflammation de la muqueuse, diminue l'abondance des selles et permet la reprise des apports énergétiques entéraux. Les titres des auto-anticorps diminuent parallèlement à la réponse clinique aux traitements médicaux. Une corticodépendance (récidive des troubles à la suite d'une diminution de la dose de corticoïde) est toutefois habituelle. Une corticorésistance (absence de réponse apparente au traitement) est également fréquente. De ce fait d'autres immunosuppresseurs puissants ont été employés. Des cas cliniques montrent l'efficacité apparente de traitements tels que la ciclosporine [40, 41], le tacrolimus [42], le sirolimus [43], le mycophénolate mofètil [44], le cyclophosphamide [45] et celle d'agents biologiques tels que l'infliximab, anticorps monoclonal chimérique anti$\mathrm{TNF} \alpha$ (facteur de nécrose tumorale $\alpha$ ) [46].
La plupart des enfants atteints répondent à une puissante immunosuppression et tolèrent finalement une alimentation entérale progressive et une réduction simultanée de la nutrition parenterale centrale. De ce fait, l'entéropathie auto-immune ne devrait pas être considérée comme une indication à une transplantation d'intestin grêle. Cette évolution clinique est très diffèrente de celle de la plupart des cas de maladie des inclusions microvillositaires, qui ne répondent pas à une immunosuppression puissante et dont le pronostic est réservé en l'absence de l'option thérapeutique que constitue la transplantation intestinale $[1,6,12]$.

Le bénéfice éventuel d'autres stratégies thérapeutiques, telles qu'une greffe de mœlle osseuse, n'est pas clairement établi chez ces enfants. Les bénéfices rapportés d'une telle décision [47] suggèrent que cette approche mérite d'être davantage envisagée dans les cas sévères ne répondant que de façon limitée ou partielle à une immunosuppression puissante et soulignent également l'importance capitale de poser un diagnostic correct devant une diarrhée prolongée chez un nourrisson ou un jeune enfant. Le regroupement des cas affectés sous la dénomination commune de «diarrhée intraitable du nourrisson» est sans intérêt et peut nuire à une prise en charge thérapeutique appropriée.

Une connaissance précise de l'étiologie d'une diarrhée prolongée est également essentielle pour le conseil génétique des familles des enfants atteints. La plupart des cas d'entéropathie auto-immune paraissent sporadiques, 
aucun autre membre de la famille n'étant affecté, les parents n'étant pas consanguins. Le syndrome IPEX lui, est lié au chromosome $\mathrm{X}$, ce qui soulève d'autres problèmes de risque dans les familles affectées, mais qui excèdent le cadre du présent article.

\section{Conclusions}

Le terme d'entéropathie auto-immune est utilisé afin de décrire une cause relativement peu fréquente de diarrhée prolongée du nourrisson. Il s'agit d'une affection inflammatoire sévère, qui répond généralement à un traitement immunosuppresseur. Il est très probable que les entéropathies auto-immunes relèvent d'un certain nombre d'étiologiques qui doivent être mieux définies afin de permettre à l'avenir des traitements mieux ciblés et un conseil génétique adéquat. Les recherches à venir devront définir les anomalies immunitaires et les processus physiopathologiques sous-jacents responsables des manifestations cliniques de la maladie. Des modèles animaux pertinents s'avèreront sans doute tout particulièrement utiles pour décrire le processus physiopathologique en cause.

Afin d'identifier les mutations génétiques à l'origine d'une entéropathie auto-immune, des fibroblastes provenant d'une biopsie cutanée devraient être immortalisés pour culture cellulaire. De même, l'ARN messager devrait être isolé de biopsies intestinales pour permettre une étude de la totalité du génome, ce qui est déjà technologiquement faisable et économiquement viable [48]. Un effort de recherche coopérative internationale devrait améliorer également les connaissances actuelles des bases moléculaires des entéropathies chroniques à médiation immunitaire.

\section{Remerciements}

P.M.S. est titulaire d'une chaire de recherche en maladies digestives au Canada. Les travaux menés au laboratoire de l'auteur sont financés par des subventions de fonctionnement des Instituts de Recherche de la Santé du Canada.

\section{Références}

1 Sherman PM, Mitchell DJ, Cutz E: Neonatal enteropathies: defining the causes of protracted diarrhea of infancy. J Pediatr Gastroenterol Nutr 2004;38:16-26.

2 Camilleri M: Chronic diarrhea: a review on pathophysiology and management for the clinical gastroenterologist. Clin Gastroenterol Hepatol 2004;2:198-206.

-3 Schiller LR: Chronic diarrhea. Gastroenterology 2004;127:287-293.

4 Bhutta ZA, Ghishan F, Lindley K, Memon IA, Mittal S, Rhoads JM: Persistent and chronic diarrhea and malabsorption: Working Group report of the second World Congress of Pediatric Gastroenterology, Hepatology, and $\mathrm{Nu}-$ trition. J Pediatr Gastroenterol Nutr 2004; 39(suppl 2):S711-S716.

5 Hill ID, Dirks MH, Liptak GS, et al: Guideline for the diagnosis and treatment of celiac disease in children: recommendations of the North American Society for Pediatric Gastroenterology, Hepatology and Nutrition. J Pediatr Gastroenterol Nutr 2005;40:1-19.

-6 Cutz E, Rhoads JM, Drumm B, et al: Microvillus inclusion disease: an inherited defect of brush-border assembly and differentiation. N Engl J Med 1989;320:646-651.

7 Phillips AD, Schmitz J: Familial microvillous atrophy: a clinicopathological survey of 23 cases. J Pediatr Gastroenterol Nutr 1992;14: 380-396.

\section{Reifen RM, Cutz E, Griffiths AM, et al: Tufting enteropathy: a newly recognized clinicopatho- logical entity associated with refractory diar- rhea in infants. J Pediatr Gastroenterol Nutr 1994;18:379-385.}

9 Patey N, Scoazec JY, Cuenod-Jabri B, et al: Distribution of cell adhesion molecules in infants with intestinal epithelial dysplasia (tufting enteropathy). Gastroenterology 1997;113: 833-843.

10 Pirisi-Hauck NC, Foss H-D, Baier J, Kurunczi S: Simultaneous occurrence of autoimmune enteropathy and recurrent deep vein thrombosis. J Pediatr Gastroenterol Nutr 2000;30:324329.

11 Carroccio A, Volta U, Di Prima L, et al: Autoimmune enteropathy and colitis in an adult patient. Dig Dis Sci 2003;48:1600-1606.

12 Cutz E, Sherman PM, Davidson GP: Enteropathies associated with protracted diarrhea of infancy: clinicopathological features, cellular and molecular mechanisms. Pediatr Pathol Lab Med 1997;17:335-368.

13 Murch SH, Fertleman CR, Rodrigues C, et al: Autoimmune enteropathy with distinct mucosal features in T-cell activation deficiency: the contribution of $\mathrm{T}$ cells to the mucosal lesion. $\mathrm{J}$ Pediatr Gastroenterol Nutr 1999;28:393399.

14 Cuenod B, Brousse N, Goulet O, et al: Classification of intractable diarrhea of infancy using clinical and immunohistological criteria. Gastroenterology 1990;99:1037-1043.
15 King C, Lic A, Koelsch K, Sarvetnick N: Homeostatic expansion of $\mathrm{T}$ cells during immune insufficiency generates autoimmunity. Cell 2004; 117:265-277.

16 Mirakian R, Richardson A, Milla PJ, et al: Protracted diarrhoea of infancy: evidence in support of an autoimmune variant. Br Med J (Clin Res Ed) 1986;293:1132-1136.

17 Colletti RB, Guillot AP, Rosen S, et al: Autoimmune enteropathy and nephropathy with circulating anti-epithelial cell antibodies. J Pediatr 1991;118:858-864.

18 Kobayashi I, Imamura K, Yamada M, et al. A 75-kD autoantigen recognized by sera from patients with X-linked autoimmune enteropathy associated with nephropathy. Clin Exp Immunol 1998;111:527-531.

$\checkmark 19$ Kobayashi I, Imamura K, Kubota M, et al: Identification of an autoimmune enteropathyrelated 75-kilodalton antigen. Gastroenterology 1999;117:823-830.

20 Ishikawa S, Kobayashi I, Hamada J-I, et al: Interaction of $\mathrm{MCC} 2$, a novel homologue of MCC tumor suppressor, with PDZ-domain protein AIE-75. Gene 2001;267:101-110.

21 Martin-Villa JM, Regueiro JR, De Juan D, et al: T-lymphocyte dysfunctions occurring together with apical gut epithelial cell autoantibodies. Gastroenterology 1991;101:390-397.

22 Moore L, Xu X, Davidson G, et al: Autoimmune enteropathy with anti-goblet cell antibodies. Hum Pathol 1995;26:1162-1168. 
-23 Shaoul R, Hong D, Okada Y, Cutz E, Marcon M: Lineage development in a patient without goblet, Paneth and enteroendocrine cells, a clue for intestinal epithelial differentiation. Pediatr Res 2005;58:492-498.

24 Yang Q, Berminham A, Finegold MJ, et al: Requirement of Math1 for secretory cell lineage commitment in the mouse intestine. Science 2001;294:2155-2158

25 Goulet O, Seidman E: Gastrointestinal manifestations of immunodeficiency. 1. Primary immunodeficiency diseases; in Walker W, Goulet O, Kleinman R, Sherman P, Shneider B, Sanderson I (eds): Pediatric Gastrointestinal Disease, ed 4. Philadelphia, B.C. Decker, 2004, pp 707-733.

26 Arniaz-Villena A, Timon M, Corell A, et al: Primary immunodeficiency caused by mutations in the gene encoding the CD3-gamma subunit of the T-lymphocyte receptor. N Engl J Med 1992;327:529-533.

27 Kaitila I, Savilahti E, Ormala T: Autoimmune enteropathy in Schimke immunoosseous dysplasia. Am J Med Genet 1998;77:427-430.

-28 Stormon M, Friedman J, King S, Cutz E, Furuya $\mathrm{KN}$ : An unusual case of diarrhea in Schimke immuno-osseous dysplasia. J Pediatr Gastroenterol Nutr 2002;35:369-371.

29 Bennett CL, Christie J, Ramsdell F, et al: The immune dysregulation, polyendocrinopathy, enteropathy, X-linked syndrome (IPEX) is caused by mutations of FOXP3. Nat Genet 2001;27:20-21.

30 Ochs HD, Ziegler SF, Torgerson TR: FOXP3 acts as a rheostat of the immune response. Immunol Rev 2005;203:156-164.
-31 Hori S, Nomura T, Sakaguchi S: Control of regulatory $\mathrm{T}$ cell development by the transcription factor FOXP3. Science 2003;299:10571061

- 32 Hori S, Sakaguchi S: Foxp3: a critical regulator of the development and function of regulatory T cells. Microbes Infect 2004;6:745-751.

33 Fontenot JD, Gavin MA, Rudensky AY: FOXP3 programs the development and function of $\mathrm{CD} 4+\mathrm{CD} 25+$ regulatory $\mathrm{T}$ cells. Nat Immunol 2003;4:330-336.

34 Bakke AC, Purtzer MZ, Wildin RS: Prospective immunological profiling in a case of immune dysregulation, polyendocrinopathy, enteropathy, X-linked syndrome (IPEX). Clin Exp Immunol 2004;137:373-378.

35 Eisenbarth GS, Gottlieb PA: Autoimmune polyendocrine syndromes. N Engl J Med 2004 350:2068-2079.

36 Ruemmele FM, Brousse N, Goulet O: Autoimmune enteropathy: molecular concepts. Curr Opin Gastroenterol 2004;20:587-591.

37 Nieves DS, Phipps RP, Pollock SJ, et al: Dermatologic and immunologic findings in the immune dysregulation, polyendocrinopathy, enteropathy, X-linked syndrome. Arch Dermatol 2004; 140:466-472.

38 Patel DD: Escape from tolerance in the human $\mathrm{X}$-linked autoimmunity-allergic disregulation syndrome and the Scurfy mouse. J Clin Invest 2001;107:155-157.

39 Lo CW, Walker WA: Chronic protracted diarrhea of infancy: a nutritional disease. Pediatrics 1983;72:786-800.

40 Seidman EG, Lacaille F, Russo P, et al: Successful treatment of autoimmune enteropathy with cyclosporine. J Pediatr 1990;117:929932.
41 Sanderson IR, Phillips AD, Spencer J, WalkerSmith JA: Response to autoimmune enteropathy to cyclosporin A therapy. Gut 1991;32: 1421-1425.

42 Bousvaros A, Leichtner AM, Book L, et al: Treatment of pediatric autoimmune enteropathy with tacrolimus (FK506). Gastroenterology 1996;111:237-243.

43 Bindl L, Torgerson T, Perroni L, Youssef N, Ochs HD, Goulet O, Ruemmele FM: Successful use of the new immune-suppressor sirolimus in IPEX (immune dysregulation, polyendocrinopathy, enteropathy, X-linked syndrome). J Pediatr 2005;147:256-259.

44 Quiroz-Tejeira RE, Ament ME, Vargas JH: Induction of remission in a child with autoimmune enteropathy using mycophenolate mofetil. J Pediatr Gastroenterol Nutr 2003;36: 482-485.

45 Oliva-Hemper MM, Loeb DM, Abraham SC, et al: Remission of severe autoimmune enteropathy after treatment with high-dose cyclophosphamide. J Pediatr Gastroenterol Nutr 2003;36:639-643.

46 Vanderhoof JA, Young RJ: Autoimmune enteropathy in a child: response to infliximab therapy. J Pediatr Gastroenterol Nutr 2002;34: 312-316.

47 Baud O, Goulet O, Canioni D, et al: Treatment of the immune dysregulation, polyendocrinopathy, enteropathy, X-linked sydrome (IPEX) by allogeneic bone marrow transplantation. $\mathrm{N}$ Engl J Med 2001;344:1758-1762.

-48 Collins SM, McHugh K, Croitoru K, et al: The establishment of a national tissue bank for inflammatory bowel disease research in Canada. Can J Gastroenterol 2003;17:107-109. 